\title{
On the Poisson Representation of a Function Harmonic in the Upper Half-Plane
}

\author{
Seçil Gergün and Iossif V. Ostrovskii
}

(Communicated by Stephan Ruscheweyh)

\begin{abstract}
New conditions for the validity of the Poisson representation (in usual and generalized form) for a function harmonic in the upper half-plane are obtained. These conditions differ from known ones by weaker growth restrictions inside the half-plane and stronger restrictions on the behavior on the real axis.
\end{abstract}

Keywords. Compactness, Green function, Nevanlinna formula, PhragménLindelöf Principle, Poisson integral, subharmonic function.

2000 MSC. 31A05, 31A10.

\section{Introduction and statement of results}

In the theory of entire and subharmonic functions the Poisson representation

$$
u(z)=\frac{y}{\pi} \int_{-\infty}^{\infty} \frac{d \nu(t)}{(x-t)^{2}+y^{2}}+c y, \quad z=x+i y, y>0,
$$

of a real-valued harmonic function $u$ in $\mathbb{C}_{+}:=\{z \in \mathbb{C}: \operatorname{Im} z>0\}$ is very important. Here, $c$ is a real constant and $\nu$ is a real-valued $\sigma$-finite Borel measure on $\mathbb{R}$ such that

$$
\int_{-\infty}^{\infty} \frac{d|\nu|(t)}{1+t^{2}}<\infty
$$

We mention applications of this representation to the theory of integral transforms $[1$, Ch. 4$]$, to the theory of entire functions $[9$, Part II], $[10$, Ch. 5$],[8$, Ch. 3], and to the theory of $H_{p}$ spaces [7, Ch. 6].

It is well-known (see, e.g. [7, p. 107], [9, p. 100]) that (1) is true if and only if $u$ can be represented in the form $u=u_{1}-u_{2}$ where $u_{1}$ and $u_{2}$ are non-negative harmonic functions in $\mathbb{C}_{+}$. Nevertheless, for several applications (see, e.g. [8, Ch. 3], [10, Ch. 5]) conditions that can be expressed in terms of the growth of $u$ are more useful. For a function $u$ continuous in the closure $\overline{\mathbb{C}}_{+}:=\{z \in \mathbb{C}: \operatorname{Im} z \geq 0\}$

Received November 27, 2001. 
of $\mathbb{C}_{+}$the strongest condition of this kind is contained in the following result of R. Nevanlinna [11].

Theorem A $([11])$. Let $u$ be a real-valued function harmonic in $\mathbb{C}_{+}$and continuous in $\overline{\mathbb{C}}_{+}$. If

(A1) there exists a sequence $\left\{r_{k}\right\}$ with $r_{k} \rightarrow \infty$ such that

$$
\int_{0}^{\pi} u^{+}\left(r e^{i \theta}\right) \sin \theta d \theta=O(r) \quad \text { for } r=r_{k} \rightarrow \infty
$$

and if

(A2)

$$
\int_{-\infty}^{\infty} \frac{u^{+}(t)}{1+t^{2}} d t<\infty
$$

then $u$ admits the representation (1) with $d \nu(t)=u(t) d t$.

In [3] and [4] different conditions for the validity of the representation (1) have been found and applied to generalizations of the Titchmarsh Convolution Theorem.

Theorem B ([3], [4]). Let $u$ be a real-valued function harmonic in $\mathbb{C}_{+}$. If

(B1) there exists a sequence $\left\{r_{k}\right\}$ with $r_{k} \rightarrow \infty$ such that

$$
\int_{0}^{\pi} u^{+}\left(r e^{i \theta}\right) \sin \theta d \theta \leq \exp (o(r)), \quad \text { for } r=r_{k} \rightarrow \infty
$$

and if

(B2) there exists a number $H>0$ such that

$$
\sup _{0<s<H} \int_{-\infty}^{\infty} \frac{|u(t+i s)|}{1+t^{2}} d t<\infty
$$

then (1) holds. If $u$ is continuous in $\overline{\mathbb{C}}_{+}$then $d \nu(t)=u(t) d t$.

Comparing conditions of Theorems $\mathrm{A}$ and $\mathrm{B}$, we see that (A1) is more restrictive than (B1) whereas (A2) is less restrictive than (B2). It should also be mentioned that continuity of $u$ in $\overline{\mathbb{C}}_{+}$is not assumed in Theorem $\mathrm{B}$.

Assumptions (B1) and (B2) in Theorem B are sharp in the following sense: The example $u(z)=\operatorname{Re}(\cos z)$ shows that "o" cannot be replaced by "O" in (B1). Moreover, (B2) cannot be replaced by

$$
\int_{-\infty}^{\infty} \frac{|u(t+i H)|}{1+t^{2}} d t<\infty
$$

for some $H>0$, as the example $u(z)=\operatorname{Im}\left((z-i H)^{2 n}\right), n \in \mathbb{N}$, shows. It is also worth mentioning that $|u(t+i s)|$ cannot be replaced with $u^{+}(t+i s)$ in (B2), as the example $u(z)=-\operatorname{Re}\left(z^{2 n}\right), n \in \mathbb{N}$, shows. 
In the present work we discuss conditions for the validity of a more general representation which includes (1) as a special case. This representation has the form

$$
u(z)=\int_{-\infty}^{\infty} P_{q}(z, t) d \nu(t)+\operatorname{Im} P(z) .
$$

Here $P_{q}(z, t)$ is the generalized Poisson kernel defined by the formula

$$
P_{q}(z, t)=\operatorname{Im}\left(\frac{1}{\pi} \frac{(1+t z)^{q}}{(t-z)\left(1+t^{2}\right)^{q}}\right), \quad q \in \mathbb{N} \cup\{0\},
$$

the measure $\nu$ is a $\sigma$-finite Borel measure on $\mathbb{R}$ satisfying

$$
\int_{-\infty}^{\infty} \frac{d|\nu|(t)}{1+|t|^{q+1}}<\infty
$$

and $P$ is a real polynomial of degree at most $q$.

Throughout the text we assume that all harmonic functions and Borel measures are real-valued.

For a function $u$ harmonic in $\mathbb{C}_{+}$and continuous in $\overline{\mathbb{C}}_{+}$which satisfies the conditions

$$
\begin{aligned}
\int_{0}^{\pi} u^{+}\left(r e^{i \theta}\right) \sin \theta d \theta & =O\left(r^{q}\right) \quad \text { as } r \rightarrow \infty \\
\int_{-\infty}^{\infty} \frac{u^{+}(t)}{1+|t|^{q+1}} d t & <\infty
\end{aligned}
$$

the representation $(2)$ (with $d \nu(t)=u(t) d t$ ) is due to R. Nevanlinna [11]. Without the continuity assumption on $u$ in $\overline{\mathbb{C}}_{+}$but with the growth condition

$$
\max _{0<\theta<\pi} u^{+}\left(r e^{i \theta}\right)=O\left(r^{\alpha}\right) \quad \text { for some } \alpha<q,
$$

it is due to N. V. Govorov [6, p. 25].

Our first result is as follows.

Theorem 1. Let $u$ be a function harmonic in $\mathbb{C}_{+}$.

If $u$ satisfies condition (B1) of Theorem $B$ and if there exists a number $\alpha>0$ such that

$$
\liminf _{s \rightarrow 0^{+}} \int_{-\infty}^{\infty} \frac{|u(t+i s)|}{1+|t|^{\alpha}} d t<\infty
$$

then $u$ admits the representation (2) with $q=\max \{n \in \mathbb{N} \cup\{0\}: n<\alpha\}$, with a $\sigma$-finite Borel measure $\nu$ on $\mathbb{R}$ satisfying

$$
\int_{-\infty}^{\infty} \frac{d|\nu|(t)}{1+|t|^{\alpha}}<\infty
$$

and with a real polynomial $P$ of degree at most $q$. 
Note that Nevanlinna's [11] and Govorov's [6] results mentioned above are not contained in Theorem 1 because neither (3) nor (4) imply (5).

It is easy to see that Theorem B is contained in Theorem 1: If $\alpha=2$ then $q=1$, $P_{1}(z, t)$ is the usual Poisson kernel, and condition (5) with $\alpha=2$ is less restrictive than (B2).

Our next result is related to the following question: Can we replace (5) with a condition requiring convergence of the integrals in (5) only over two horizontal lines? In some sense, there is an affirmative answer to this question. To formulate this more precisely, we need a lemma.

Lemma 1. Let $u$ be a function harmonic in $\mathbb{C}_{+}$.

If there exists a number $H>0$ such that for all $R>0$

$$
\sup _{0<y<H} \int_{-R}^{R}|u(x+i y)| d x<\infty
$$

then there exists a Borel measure $\nu$ on $\mathbb{R}$ with $|\nu|([-R, R])<\infty$ for all $R>0$ and such that the function

$$
u(z)-\int_{-R}^{R} P_{q}(z, t) d \nu(t), \quad q \in \mathbb{N} \cup\{0\},
$$

is harmonic in $\mathbb{C}_{+}$, continuous in $\mathbb{C}_{+} \cup(-R, R)$, and vanishes on $(-R, R)$.

Now we are ready to state our second result.

Theorem 2. Let $u$ be a function harmonic in $\mathbb{C}_{+}$satisfying condition (6) of Lemma 1 and condition (B1) of Theorem B.

If there exist numbers $H>0$ and $\alpha>0$ such that

$$
\int_{-\infty}^{\infty} \frac{|u(t+i H)|}{1+|t|^{\alpha}} d t+\int_{-\infty}^{\infty} \frac{d|\nu|(t)}{1+|t|^{\alpha}}<\infty,
$$

where $\nu$ is the $\sigma$-finite Borel measure defined in Lemma 1 then $u$ admits the representation (2) with $q$ and $P$ as in Theorem 1.

The following corollary to Theorem 2 is immediate.

Corollary 1. Let $u$ be a function harmonic in $\mathbb{C}_{+}$and continuous in $\overline{\mathbb{C}}_{+}$which satisfies condition (B1) of Theorem B.

If there exist numbers $H>0$ and $\alpha>0$ such that

$$
\int_{-\infty}^{\infty} \frac{|u(t)|+|u(t+i H)|}{1+|t|^{\alpha}} d t<\infty
$$

then $u$ admits the representation (2) with $d \nu(t)=u(t) d t$ and with $q$ and $P$ as in Theorem 1. 
The rest of the paper is organized as follows: In Section 2 we prove some auxiliary results. Section 3 is devoted to the proof of a weaker version of Theorem 1 where condition (5) is replaced by a stronger one. Section 4 is devoted to the proof of Lemma 1. In Section 5 we prove Theorem 2. Finally, using the result of Section 3 and Theorem 2, we prove Theorem 1 in Section 6 .

\section{Auxiliary results}

Lemma 2. The generalized Poisson kernel $P_{q}(z, t)$ satisfies the estimate

(9) $\left|P_{q}(z, t)\right| \leq \frac{y}{|t-z|^{2}}\left(A_{q} \frac{(1+|z|)^{q-1}}{(1+|t|)^{q-1}}+B_{q} \frac{(1+|z|)^{q}}{(1+|t|)^{q}}\right), \quad z=x+i y \in \mathbb{C}_{+}$, where $A_{q}$ and $B_{q}$ are nonnegative constants.

Proof. For $q=0,1$ the inequality is trivial (with the choice $A_{0}=0, B_{0}=1 / \pi$, $A_{1}=1 / \pi$ and $\left.B_{1}=0\right)$. Hence, we may assume $q \geq 2$. We have

$$
\begin{aligned}
P_{q}(z, t) & =\frac{\operatorname{Im}\left((t-\bar{z})(1+t z)^{q}\right)}{\pi|t-z|^{2}\left(1+t^{2}\right)^{q}} \\
\operatorname{Im}\left((t-\bar{z})(1+t z)^{q}\right) & =\operatorname{Im}\left((t-\bar{z}) \sum_{k=0}^{q}\left(\begin{array}{l}
q \\
k
\end{array}\right) t^{k} z^{k}\right) \\
& =\sum_{k=0}^{q}\left(\begin{array}{l}
q \\
k
\end{array}\right) t^{k+1} \operatorname{Im}\left(z^{k}\right)-\sum_{k=0}^{q}\left(\begin{array}{l}
q \\
k
\end{array}\right) t^{k}|z|^{2} \operatorname{Im}\left(z^{k-1}\right) \\
& =: S_{1}+S_{2} .
\end{aligned}
$$

Using the inequality $|\sin k \theta| \leq k \sin \theta, 0 \leq \theta \leq \pi, k \in \mathbb{N}$, we obtain

$$
\begin{aligned}
& \left|S_{1}\right| \leq \sum_{k=1}^{q} k\left(\begin{array}{l}
q \\
k
\end{array}\right)|t|^{k+1}|z|^{k-1} y \leq q(1+|t|)^{q+1}(1+|z|)^{q-1} y, \\
& \left|S_{2}\right| \leq y+\sum_{k=2}^{q}(k-1)\left(\begin{array}{l}
q \\
k
\end{array}\right)|t|^{k}|z|^{k} y \leq q(1+|t|)^{q}(1+|z|)^{q} y .
\end{aligned}
$$

From (10) and (11) we get

$$
\left|P_{q}(z, t)\right| \leq \frac{2^{q} q}{\pi} \frac{y}{|t-z|^{2}} \frac{(1+|z|)^{q-1}((1+|t|)+(1+|z|))}{(1+|t|)^{q}} .
$$

We also need the following immediate corollary to Lemma 2.

Corollary 2. The generalized Poisson kernel $P_{q}(z, t)$ satisfies the estimate

$$
\left|P_{q}(z, t)\right| \leq C_{q} \frac{y}{|t-z|^{2}} \frac{(1+|z|)^{q}}{(1+|t|)^{q-1}}, \quad z=x+i y \in \mathbb{C}_{+},
$$


where $C_{q}$ is a positive constant.

Lemma 3. Let $u$ be a function harmonic in $\mathbb{C}_{+}$.

If there exist numbers $H>0$ and $\alpha>0$ such that

$$
\sup _{0<s<H} \int_{-\infty}^{\infty} \frac{|u(t+i s)|}{1+|t|^{\alpha}}<\infty
$$

then $u$ admits the representation

$$
u(z)=\int_{-\infty}^{\infty} P_{q}(z, t) d \nu(t)+U(z)
$$

with $q$ and $\nu$ as in Theorem 1 and with a function $U$ harmonic in $\mathbb{C}$ such that $U(x)=0$ for $x \in \mathbb{R}$.

Proof. Consider the family of Borel measures on $\mathbb{R}$

$$
\sigma_{s}(E)=\int_{E} \frac{u(t+i s)}{1+|t|^{\alpha}} d t, \quad 0<s<H .
$$

By (13), each sequence $\left\{\sigma_{s_{k}}\right\}$ with $\lim _{k \rightarrow \infty} s_{k}=0$ contains a subsequence (which we also denote by $\left\{\sigma_{s_{k}}\right\}$ ) which is weak-star convergent to a finite Borel measure $\sigma$ on $\overline{\mathbb{R}}$ (the 2-point compactification of $\mathbb{R}$ ). Hence, noting that

$$
\lim _{t \rightarrow \pm \infty}\left(1+|t|^{\alpha}\right) P_{q}(z, t)=\left\{\begin{array}{cl}
0 & \text { if } \alpha<q+1 \\
\frac{( \pm 1)^{q+1}}{\pi} \operatorname{Im}\left(z^{q}\right) & \text { if } \alpha=q+1
\end{array},\right.
$$

we get

$$
\begin{aligned}
& \lim _{k \rightarrow \infty} \int_{-\infty}^{\infty} u\left(t+i s_{k}\right) P_{q}(z, t) d t \\
& =\int_{-\infty}^{\infty}\left(1+|t|^{\alpha}\right) P_{q}(z, t) d \sigma(t) \quad \text { if } \alpha<q+1, \\
& \lim _{k \rightarrow \infty} \int_{-\infty}^{\infty} u\left(t+i s_{k}\right) P_{q}(z, t) d t \\
& =\int_{-\infty}^{\infty}\left(1+|t|^{\alpha}\right) P_{q}(z, t) d \sigma(t) \\
& +\frac{1}{\pi} \operatorname{Im}\left(z^{q}\right)\left[\sigma(\{\infty\})+(-1)^{q+1} \sigma(\{-\infty\})\right] \quad \text { if } \alpha=q+1 .
\end{aligned}
$$

Joining (15) and (16) and setting $d \nu(t)=\left(1+|t|^{\alpha}\right) d \sigma(t)$ we obtain

$$
\lim _{k \rightarrow \infty} \int_{-\infty}^{\infty} u\left(t+i s_{k}\right) P_{q}(z, t) d t=\int_{-\infty}^{\infty} P_{q}(z, t) d \nu(t)+A \operatorname{Im}\left(z^{q}\right),
$$

for some constant $A$. 
Consider the family of functions

$$
\begin{array}{r}
U_{s}(z)=u(z+i s)-\int_{-\infty}^{\infty} u(t+i s) P_{q}(z, t) d t, \\
z=x+i y \in \mathbb{C}_{+}, 0<s<\frac{H}{2} .
\end{array}
$$

From (13) it follows that for each $s$ with $0<s<H / 2$ the function $U_{s}$ is harmonic in $\mathbb{C}_{+}$and becomes continuous in $\overline{\mathbb{C}}_{+}$if we set $U_{s}(x)=0$ for $x \in \mathbb{R}$. By the Symmetry Principle it can be extended harmonically to $\mathbb{C}$ and then fulfills $U_{s}(z)=-U_{s}(\bar{z})$ for $z \in \mathbb{C}$.

Now let us show that, for any fixed $R$, the family $\left\{U_{s}: 0<s<H / 2\right\}$ is uniformly bounded in the rectangle $\Pi_{R}=\{z \in \mathbb{C}:|\operatorname{Re} z| \leq R,|\operatorname{Im} z| \leq H / 4\}$. To show this, we shall first show that

$$
\int_{-\infty}^{\infty} \frac{\left|U_{s}(x+i y)\right|}{1+|x|^{q+1}} d x \leq C \quad \text { for }|y| \leq \frac{H}{2},
$$

for some constant ${ }^{1} C$ which does not depend on $y$ and $s$.

Note that it is enough to show (19) for $0<y \leq H / 2$. Using Fubini's Theorem and (18) we get

$$
\begin{aligned}
& \int_{-\infty}^{\infty} \frac{\left|U_{s}(x+i y)\right|}{1+|x|^{q+1}} d x \\
& \quad \leq \int_{-\infty}^{\infty} \frac{|u(x+i(y+s))|}{1+|x|^{q+1}} d x+\int_{-\infty}^{\infty}|u(t+i s)|\left(\int_{-\infty}^{\infty} \frac{\left|P_{q}(z, t)\right|}{1+|x|^{q+1}} d x\right) d t \\
& \quad=: I_{1}+I_{2} .
\end{aligned}
$$

By (13), $I_{1}$ is bounded by a constant not depending on $y$ and $s$. From (9) we obtain for $0<y \leq H / 2$

$$
\begin{aligned}
I_{2} \leq & A_{q} \int_{-\infty}^{\infty} \frac{|u(t+i s)|}{(1+|t|)^{q-1}}\left(\int_{-\infty}^{\infty} \frac{y}{(x-t)^{2}+y^{2}} \frac{(1+|z|)^{q-1}}{(1+|x|)^{q+1}} d x\right) d t \\
& +B_{q} \int_{-\infty}^{\infty} \frac{|u(t+i s)|}{(1+|t|)^{q}}\left(\int_{-\infty}^{\infty} \frac{y}{(x-t)^{2}+y^{2}} \frac{(1+|z|)^{q}}{(1+|x|)^{q+1}} d x\right) d t \\
\leq & A_{q, H} \int_{-\infty}^{\infty} \frac{|u(t+i s)|}{(1+|t|)^{q-1}}\left(\int_{-\infty}^{\infty} \frac{y}{(x-t)^{2}+y^{2}} \frac{1}{1+x^{2}} d x\right) d t \\
& +B_{q, H} \int_{-\infty}^{\infty} \frac{|u(t+i s)|}{(1+|t|)^{q}}\left(\int_{-\infty}^{\infty} \frac{y}{(x-t)^{2}+y^{2}} \frac{1}{1+|x|} d x\right) d t .
\end{aligned}
$$

\footnotetext{
${ }^{1}$ Here and in what follows the letters $A, B, C$ and $D$ with or without subscripts denote positive constants.
} 
Since

$$
\int_{-\infty}^{\infty} \frac{y}{(x-t)^{2}+y^{2}} \frac{1}{1+x^{2}} d x=\frac{\pi(y+1)}{t^{2}+(y+1)^{2}} \leq \frac{\pi(H+2)}{2\left(t^{2}+1\right)},
$$

the Schwarz inequality gives

$$
\begin{aligned}
\int_{-\infty}^{\infty} & \frac{y}{(x-t)^{2}+y^{2}} \frac{1}{1+|x|} d x \\
& \leq\left(\int_{-\infty}^{\infty} \frac{y}{(x-t)^{2}+y^{2}} \frac{1}{1+x^{2}} d x\right)^{1 / 2}\left(\int_{-\infty}^{\infty} \frac{y}{(x-t)^{2}+y^{2}} d x\right)^{1 / 2} \\
& \leq \frac{\pi \sqrt{H+2}}{1+|t|}
\end{aligned}
$$

Inserting (22) and (23) into (21) and using (13) we conclude that $I_{2}$ is bounded by a constant not depending on $y$ and $s$. This proves (19).

Since $\left|U_{s}\right|$ is subharmonic, we have for any $\rho>0$

$$
\begin{aligned}
\left|U_{s}(z)\right| & \leq \frac{1}{\pi \rho^{2}} \iint_{|\xi+i \eta-z| \leq \rho}\left|U_{s}(\xi+i \eta)\right| d \xi d \eta \\
& =\frac{1}{\pi \rho^{2}} \iint_{|\xi+i \eta-z| \leq \rho}\left(1+|\xi|^{q+1}\right) \frac{\left|U_{s}(\xi+i \eta)\right|}{1+|\xi|^{q+1}} d \xi d \eta \\
& \leq \frac{1+(|z|+\rho)^{q+1}}{\pi \rho^{2}} \int_{\operatorname{Im}(z)-\rho}^{\operatorname{Im}(z)+\rho}\left(\int_{\operatorname{Re}(z)-\rho}^{\operatorname{Re}(z)+\rho} \frac{\left|U_{s}(\xi+i \eta)\right|}{1+|\xi|^{q+1}} d \xi\right) d \eta
\end{aligned}
$$

With the choice $\rho=H / 4$ and by the aid of (19) we obtain for $z \in \Pi_{R}$

$$
\begin{aligned}
\left|U_{s}(z)\right| & \leq \frac{1+\left(R+\frac{H}{2}\right)^{q+1}}{\pi\left(\frac{H}{4}\right)^{2}} \int_{-H / 2}^{H / 2}\left(\int_{-\infty}^{\infty} \frac{\left|U_{s}(\xi+i \eta)\right|}{1+|\xi|^{q+1}} d \xi\right) d \eta \\
& \leq \frac{1+\left(R+\frac{H}{2}\right)^{q+1}}{\pi\left(\frac{H}{4}\right)^{2}} H C .
\end{aligned}
$$

Thus the family $\left\{U_{s}: 0<s<H / 2\right\}$ is uniformly bounded in $\Pi_{R}$.

Let $\left\{s_{k}\right\}_{k=1}^{\infty}$ be a sequence such that (17) holds. By the well-known Compactness Principle for harmonic functions we can extract a subsequence (which we also denote by $\left.\left\{s_{k}\right\}\right)$ such that the sequence $\left\{U_{s_{k}}\right\}_{k=1}^{\infty}$ is uniformly convergent on any compact subset of the strip $\{z \in \mathbb{C}:|\operatorname{Im} z|<H / 4\}$. Let $U$ be the limiting function. Evidently $U$ is harmonic in this strip and $U(x)=0$ for $x \in \mathbb{R}$. With the choice $s=s_{k}$ in (18) and taking limit as $k \rightarrow \infty$ we obtain

$$
U(z)=u(z)-\int_{-\infty}^{\infty} P_{q}(z, t) d \nu(t)-A \operatorname{Im}\left(z^{q}\right),
$$

for $0<\operatorname{Im} z<H / 4$. The right hand side of $(25)$ is a harmonic function in $\mathbb{C}_{+}$. Therefore $U$ can be extended harmonically to $\mathbb{C}_{+}$. Since $U(x)=0$ for $x \in \mathbb{R}$, 
this function can be extended harmonically to $\mathbb{C}$. To derive formula (14) we only have to replace $U(z)+A \operatorname{Im}\left(z^{q}\right)$ by $U(z)$.

\section{A weaker version of Theorem 1}

Theorem 1'. Let $u$ be a function harmonic in $\mathbb{C}_{+}$, satisfying condition (B1) of Theorem $B$ and condition (13). Then the assertion of Theorem 1 holds.

Proof. By Lemma 3 the function $u$ admits the representation (14). Our aim is to show $U(z)=\operatorname{Im} P(z)$ where $P$ is a real polynomial of degree at most $q$. The proof of this assertion is obtained in several steps.

Step 1: We show that

$$
\int_{0}^{\pi} U^{+}\left(r e^{i \theta}\right) \sin \theta d \theta \leq \exp (o(r)) \quad \text { for } r=r_{k} \rightarrow \infty .
$$

From (14) we get

$$
\begin{aligned}
& \int_{0}^{\pi} U^{+}\left(r e^{i \theta}\right) \sin \theta d \theta \\
& \quad \leq \int_{0}^{\pi} u^{+}\left(r e^{i \theta}\right) \sin \theta d \theta+\int_{0}^{\pi} \sin \theta\left(\int_{-\infty}^{\infty}\left|P_{q}\left(r e^{i \theta}, t\right)\right| d|\nu|(t)\right) d \theta .
\end{aligned}
$$

The first integral on the right hand side can be estimated by (B1). Let us denote the second integral by $I$. Using Fubini's Theorem, the relation

$$
\int_{0}^{\pi} \frac{\sin ^{2} \varphi d \varphi}{r^{2}+t^{2}-2 r t \cos \varphi}=\frac{\pi}{2} \min \left\{\frac{1}{r^{2}}, \frac{1}{t^{2}}\right\} \leq \frac{\pi}{1+t^{2}} \quad \text { for } r \geq 1
$$

and estimate (12) we obtain

$$
\begin{aligned}
I & \leq D_{q} r^{q+1} \int_{-\infty}^{\infty} \frac{1}{(1+|t|)^{q-1}}\left(\int_{0}^{\pi} \frac{\sin ^{2} \theta}{t^{2}-2 t r \cos \theta+r^{2}} d \theta\right) d|\nu|(t) \\
& \leq D_{q} r^{q+1} \int_{-\infty}^{\infty} \frac{1}{(1+|t|)^{q-1}} \frac{\pi}{1+t^{2}} d|\nu|(t)=O\left(r^{q+1}\right) \quad \text { as } r \rightarrow \infty .
\end{aligned}
$$

This implies (26).

Step 2: Now we show that

$$
\int_{0}^{\pi}\left|U\left(r e^{i \theta}\right)\right| \sin \theta d \theta \leq \exp (o(r)) \quad \text { for } r=r_{k} \rightarrow \infty .
$$

By the Nevanlinna formula (see, e.g. [5, p. 16] or $[9$, p. 193]) we have

$$
U(i)=\frac{1}{2 \pi} \int_{0}^{\pi} \frac{4\left(r_{k}^{2}-1\right) r_{k} \sin \theta}{\left|r_{k} e^{i \theta}-i\right|^{2}\left|r_{k} e^{-i \theta}-i\right|^{2}} U\left(r_{k} e^{i \theta}\right) d \theta .
$$

Note that for $r_{k} \geq 2$

$$
\frac{C_{1}}{r_{k}} \sin \theta \leq \frac{4\left(r_{k}^{2}-1\right) r_{k} \sin \theta}{\left|r_{k} e^{i \theta}-i\right|^{2}\left|r_{k} e^{-i \theta}-i\right|^{2}} \leq \frac{C_{2}}{r_{k}} \sin \theta .
$$


Since $U=2 U^{+}-|U|$, we obtain

$$
\begin{aligned}
\int_{0}^{\pi}\left|U\left(r_{k} e^{i \theta}\right)\right| \sin \theta d \theta & \leq \frac{r_{k}}{C_{1}} \int_{0}^{\pi} \frac{4\left(r_{k}{ }^{2}-1\right) r_{k} \sin \theta}{\left|r_{k} e^{i \theta}-i\right|^{2}\left|r_{k} e^{-i \theta}-i\right|^{2}}\left|U\left(r_{k} e^{i \theta}\right)\right| d \theta \\
& \leq \frac{2 C_{2}}{C_{1}} \int_{0}^{\pi} U^{+}\left(r_{k} e^{i \theta}\right) \sin \theta d \theta-\frac{2 \pi r_{k}}{C_{1}} U(i) .
\end{aligned}
$$

Then (28) follows from (26).

Step 3: We show that

$$
|U(z)| \leq \exp (o(|z|)) \quad \text { for }|z|=\frac{r_{k}}{2} \rightarrow \infty .
$$

Since $U(z)=-U(\bar{z})$ for $z \in \mathbb{C}$, it is enough to prove (29) only for $z \in \mathbb{C}_{+}$. By a further application of the Nevanlinna formula we obtain

$$
\begin{array}{r}
U(z)=\frac{1}{2 \pi} \int_{0}^{\pi} \frac{\left(r_{k}^{2}-\frac{r_{k}{ }^{2}}{4}\right) 4 r_{k}\left(\frac{r_{k}}{2}\right) \sin \theta \sin \varphi}{\left|r_{k} e^{i \theta}-\left(\frac{r_{k}}{2}\right) e^{i \varphi}\right|^{2}\left|r_{k} e^{-i \theta}-\left(\frac{r_{k}}{2}\right) e^{i \varphi}\right|^{2}} U\left(r_{k} e^{i \theta}\right) d \theta \\
\text { if } z=\left(\frac{r_{k}}{2}\right) e^{i \varphi} \in \mathbb{C}_{+} .
\end{array}
$$

Simple estimates and (28) show that

$$
|U(z)| \leq \frac{12}{\pi} \int_{0}^{\pi}\left|U\left(r_{k} e^{i \theta}\right)\right| \sin \theta d \theta \leq \exp \left(o\left(r_{k}\right)\right) \quad \text { for } r_{k} \rightarrow \infty .
$$

Step 4: Now, we show that

$$
|U(z)|=o\left(|z|^{q+1}\right) \quad \text { as } z \rightarrow \infty,|\operatorname{Im} z|<\frac{H}{2} .
$$

Formula (14) implies that $U$ has a similar representation as the function $U_{s}$ in formula (18). Calculations similar to (20)-(23) show that there exists a constant $C_{q, H} \geq 0$ such that

$$
\int_{-\infty}^{\infty} \frac{|U(x+i y)|}{1+|x|^{q+1}} d x \leq C_{q, H} \quad \text { if }|y| \leq H,
$$

instead of (19), and

$$
|U(z)| \leq \frac{1+(|z|+\rho)^{q+1}}{\pi \rho^{2}} \int_{\operatorname{Im}(z)-\rho}^{\operatorname{Im}(z)+\rho}\left(\int_{\operatorname{Re}(z)-\rho}^{\operatorname{Re}(z)+\rho} \frac{|U(\xi+i \eta)|}{1+|\xi|^{q+1}} d \xi\right) d \eta,
$$

instead of (24). Putting $\rho=H / 2,|\operatorname{Im} z|<H / 2$, we get

$$
|U(z)| \leq \frac{1+(|z|+H / 2)^{q+1}}{\pi(H / 2)^{2}} \iint \frac{|U(\xi+i \eta)|}{1+|\xi|^{q+1}} d \xi d \eta
$$

where the double integral is taken over the rectangle

$$
\left\{(\xi, \eta) \in \mathbb{R}^{2}:|\xi-\operatorname{Re} z|<\frac{H}{2},|\eta|<H\right\} .
$$


This integral tends to 0 as $z \rightarrow \infty$ because its integrand is summable over the whole strip $\left\{(\xi, \eta) \in \mathbb{R}^{2}:|\eta|<H\right\}$ as seen in (31). Thus, (30) is valid.

Step 5: Let $G$ be the entire function which is determined uniquely by the conditions $\operatorname{Re} G(z)=U(z)$ and $G(0)=0$. We shall show that there exists a sequence $\left\{R_{k}\right\}, R_{k} \rightarrow \infty$, such that

$$
|G(z)| \leq \exp (o(|z|)) \quad \text { for }|z|=R_{k} \rightarrow \infty
$$

and that

$$
|G(z)|=o\left(|z|^{q+2}\right) \quad \text { for } z \rightarrow \infty,|\operatorname{Im} z| \leq \frac{H}{4} .
$$

To this end we use the Schwarz formula

$$
G(z+\zeta)=\frac{1}{2 \pi} \int_{0}^{2 \pi} U\left(z+\rho e^{i \theta}\right) \frac{\rho e^{i \theta}+\zeta}{\rho e^{i \theta}-\zeta} d \theta+i \operatorname{Im} G(z) \quad \text { if }|\zeta|<\rho .
$$

Differentiating with respect to $\zeta$ we obtain at $\zeta=0$

$$
G^{\prime}(z)=\frac{1}{\pi \rho} \int_{0}^{2 \pi} U\left(z+\rho e^{i \theta}\right) e^{-i \theta} d \theta .
$$

This implies

$$
\left|G^{\prime}(z)\right| \leq \frac{2}{\rho} \max _{|\zeta-z| \leq \rho}|U(\zeta)| .
$$

Now, choose $\rho=H / 4$. For $|z| \leq R_{k}:=r_{k} / 2-\rho$, we get from (34) and (29)

$$
\begin{aligned}
|G(z)| & =\left|\int_{0}^{z} G^{\prime}(\zeta) d \zeta\right| \leq R_{k} \max _{|z| \leq R_{k}}\left|G^{\prime}(z)\right| \\
& \leq R_{k} \exp \left(o\left(r_{k} / 2\right)\right) \leq \exp \left(o\left(R_{k}\right)\right) \quad \text { for }|z|=R_{k} \rightarrow \infty .
\end{aligned}
$$

Hence, (32) is valid.

For $|\operatorname{Im} z| \leq H / 4$ we get from (34) and (30)

$$
\left|G^{\prime}(z)\right|=o\left(|z|^{q+1}\right), \quad z \rightarrow \infty .
$$

From this, (33) follows by integration.

Step 6: We are now ready to complete the proof of Theorem 1'.

We apply the well-known version of the Phragmén-Lindelöf Principle for the half-plane (see, e.g. [12, p. 43]) to the function $G(z) /(z+i)^{q+2}$ in $\mathbb{C}_{+}$and to the function $G(z) /(z-i)^{q+2}$ in $\mathbb{C}_{-}$. This shows that (33) holds in the whole complex plane $\mathbb{C}$. But then, by Liouville's Theorem, the function $G$ is a polynomial of degree at most $q+1$. Since $\operatorname{Re} G(t)=U(t)=0$ for $t \in \mathbb{R}$ and $G(0)=0$, we have $G(z)=i a_{q+1} z^{q+1}+i a_{q} z^{q}+\cdots+i a_{1} z$ with coefficients $a_{j} \in \mathbb{R}, j=1,2, \ldots, q+1$. Hence, $U(z)=\operatorname{Im}\left(-a_{q+1} z^{q+1}-\cdots-a_{1} z\right)$. Clearly, (31) yields $a_{q+1}=0$, so that (2) holds. 


\section{Proof of Lemma 1}

We proceed in a manner similiar to the proof of Lemma 3.

Fix $R$ and consider the family of Borel measures on $[-R, R]$

$$
\nu_{R, s}(E)=\int_{E} u(t+i s) d t, \quad 0<s<H, \quad E \subset[-R, R] .
$$

Each sequence $\left\{\nu_{R, s_{k}}\right\}, \lim _{k \rightarrow \infty} s_{k}=0$, contains a subsequence (which we also denote by $\left.\left\{\nu_{R, s_{k}}\right\}\right)$ which ist weak-star convergent to a finite Borel measure $\nu_{R}$ on $[-R, R]$. Hence

$$
\lim _{k \rightarrow \infty} \int_{-R}^{R} u\left(t+i s_{k}\right) P_{q}(z, t) d t=\int_{-R}^{R} P_{q}(z, t) d \nu_{R}(t) .
$$

Consider the family of functions

$$
U_{R, s}(z)=u(z+i s)-\int_{-R}^{R} P_{q}(z, t) u(t+i s) d t, \quad z \in \mathbb{C}_{+}, \quad 0<s<\frac{H}{2} .
$$

Clearly, $U_{R, s}$ is harmonic in $\mathbb{C}_{+}$and continuous in $\mathbb{C}_{+} \cup(-R, R)$ if we define $U_{R, s}(x)=0$ for $x \in \mathbb{R}$. By the Symmetry Principle, $U_{R, s}$ can be extended to a function (which we also denote by $U_{R, s}$ ) harmonic in $\mathbb{C} \backslash[(-\infty,-R] \cup[R, \infty)]$ which satisfies $U_{R, s}(z)=-U_{R, s}(\bar{z})$ there.

Take any $R^{\prime}>R$, any $\epsilon>0$ sufficiently small and consider the compact set

$$
\begin{aligned}
\Pi_{R, R^{\prime}, \epsilon}:= & \left\{z \in \mathbb{C}:|\operatorname{Re} z| \leq R^{\prime},|\operatorname{Im} z| \leq \frac{H}{4}\right\} \\
& \backslash\{z \in \mathbb{C}:|\operatorname{Re} z|>R-\epsilon,|\operatorname{Im} z|<\epsilon\}
\end{aligned}
$$

(see Figure 1).

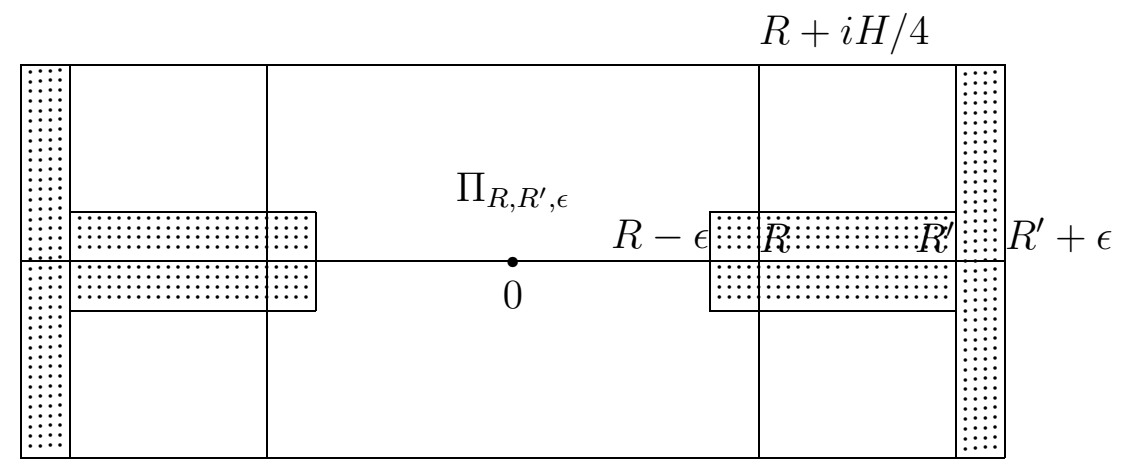

FiguRE 1

We shall show that the family $\left\{U_{R, s}: 0<s<H / 2\right\}$ is uniformly bounded on the set $\Pi_{R, R^{\prime}, \epsilon}$. 
To do so, we first prove that

$$
\sup _{0<y<H / 2} \int_{-\left(R^{\prime}+\epsilon\right)}^{\left(R^{\prime}+\epsilon\right)}\left|U_{R, s}(x+i y)\right| d x<\infty .
$$

From (36) it follows that

$$
\begin{aligned}
\int_{-\left(R^{\prime}+\epsilon\right)}^{R^{\prime}+\epsilon}\left|U_{R, s}(x+i y)\right| d x \leq & \int_{-\left(R^{\prime}+\epsilon\right)}^{R^{\prime}+\epsilon}|u(x+i(y+s))| d x \\
& +\int_{-\left(R^{\prime}+\epsilon\right)}^{R^{\prime}+\epsilon}\left(\int_{-R}^{R}\left|P_{q}(x+i y, t)\right||u(t+i s)| d t\right) d x \\
=: & I_{1}+I_{2} .
\end{aligned}
$$

Using Fubini's Theorem and estimate (12) we get

$$
\begin{aligned}
I_{2} & =\int_{-R}^{R}|u(t+i s)|\left(\int_{-\left(R^{\prime}+\epsilon\right)}^{R^{\prime}+\epsilon}\left|P_{q}(x+i y, t)\right| d x\right) d t \\
& \leq C_{q, R^{\prime}} \int_{-R}^{R}|u(t+i s)|\left(\int_{-\left(R^{\prime}+\epsilon\right)}^{R^{\prime}+\epsilon} \frac{y}{(x-t)^{2}+y^{2}} d x\right) d t \\
& \leq \pi C_{q, R^{\prime}} \int_{-R}^{R}|u(t+i s)| d t
\end{aligned}
$$

where $C_{q, R^{\prime}}$ does not depend on $y$ and $s$. Since $0<s<H / 2$, it follows from (6) that $I_{2}$ is bounded by a constant which does not depend on $y$ and $s$.

For $0<y<H / 2$ we have $0<y+s<H$ and (6) shows that $I_{1}$ is uniformly bounded. Therefore, (37) holds.

Since $\left|U_{R, s}\right|$ is subharmonic in $\mathbb{C} \backslash[(-\infty,-R] \cup[R, \infty)]$ for $z \in \Pi_{R, R^{\prime}, \epsilon}$ we obtain the estimate

$$
\begin{aligned}
\left|U_{R, s}(z)\right| & \leq \frac{1}{\pi \epsilon^{2}} \iint_{|\xi+i \eta-z| \leq \epsilon}\left|U_{R, s}(\xi+i \eta)\right| d \xi d \eta \\
& \leq \frac{1}{\pi \epsilon^{2}} \int_{-H / 2}^{H / 2} \int_{-\left(R^{\prime}+\epsilon\right)}^{R^{\prime}+\epsilon}\left|U_{R, s}(\xi+i \eta)\right| d \xi d \eta .
\end{aligned}
$$

By the aid of (37) this shows that the family $\left\{U_{R, s}: 0<s<H / 2\right\}$ is uniformly bounded on $\Pi_{R, R^{\prime}, \epsilon}$.

Now, let $\left\{s_{k}\right\}$ be a sequence such that (35) holds. By the Compactness Principle for harmonic functions we can extract a subsequence (which we also denote by $\left.\left\{s_{k}\right\}\right)$ such that the sequence $\left\{U_{R, s_{k}}\right\}$ is uniformly convergent on any compact subset of the slit strip

$$
\Pi=\left\{z:|\operatorname{Im} z|<\frac{H}{4}\right\} \backslash[(-\infty,-R] \cup[R, \infty)] .
$$


Let $U_{R}$ be the limiting function. Clearly, $U_{R}$ is harmonic in $\Pi$ and satisfies $U(x)=0$ for $x \in(-R, R)$. With the choice $s=s_{k}$ in (36) and taking limit as $k \rightarrow \infty$ we obtain

$$
U_{R}(z)=u(z)-\int_{-R}^{R} P_{q}(z, t) d \nu_{R}(t) \quad \text { for } 0<\operatorname{Im} z<\frac{H}{4} .
$$

The right hand side of $(38)$ is a harmonic function in $\mathbb{C}_{+}$. Therefore $U_{R}$ can be extended harmonically to $\mathbb{C}_{+}$. Since $U_{R}(x)=0$ for $x \in(-R, R)$, the function $U_{R}$ can further be extended harmonically to $\mathbb{C} \backslash[(-\infty,-R] \cup[R, \infty)]$.

Now, we show that for $R_{2}>R_{1}$ the restriction of $\nu_{R_{2}}$ to $\left[-R_{1}, R_{1}\right]$ coincides with $\nu_{R_{1}}$.

We have

$$
U_{R_{2}}(z)-U_{R_{1}}(z)+\int_{R_{1}<|t| \leq R_{2}} P_{q}(z, t) d \nu_{R_{2}}(t)=\int_{-R_{1}}^{R_{1}} P_{q}(z, t)\left[d \nu_{R_{1}}(t)-d \nu_{R_{2}}(t)\right] .
$$

The left-hand side is a harmonic function in $\mathbb{C} \backslash\left[\left(-\infty,-R_{1}\right] \cup\left[R_{1}, \infty\right)\right]$ vanishing on $\left(-R_{1}, R_{1}\right)$. Since

$$
P_{q}(z, t)=\frac{y}{\pi} \frac{1}{(x-t)^{2}+y^{2}}+Q(x, y, t)
$$

where $Q$ is a harmonic polynomial in $x$ and $y$ vanishing for $y=0$, we see that $\nu_{R_{2}}$ coincides with $\nu_{R_{1}}$ on $\left(-R_{1}, R_{1}\right)$.

\section{Proof of Theorem 2}

Let $\nu$ be the $\sigma$-finite Borel measure defined in Lemma 1. Since

$$
P_{q}(z, t)=O\left(t^{-q-1}\right) \quad \text { as } t \rightarrow \infty,
$$

and since $\alpha \leq q+1$, the convergence of the second integral in (7) ensures the convergence and harmonicity in $\mathbb{C}_{+}$of the integral

$$
\int_{-\infty}^{\infty} P_{q}(z, t) d \nu(t)
$$

Define

$$
U(z):=u(z)-\int_{-\infty}^{\infty} P_{q}(z, t) d \nu(t) .
$$

We shall show that $U(z)=\operatorname{Im} P(z)$ with some real polynomial $P$ of degree at most $q$.

For arbitrary $R>0$ we write

$$
U(z)=\left(u(z)-\int_{-R}^{R} P_{q}(z, t) d \nu(t)\right)-\int_{|t|>R} P_{q}(z, t) d \nu(t)=I_{1}+I_{2} .
$$

It is easy to see from Lemma 1 that $I_{1}$ is a harmonic function in the slit plane $\mathbb{C} \backslash[(-\infty,-R] \cup[R, \infty)]$ vanishing on $(-R, R)$. Using (39) we see that $I_{2}$ has this 
property, too. Hence, $U$ is continuous in $\overline{\mathbb{C}}_{+}$, if we define $U(x):=0$ for $x \in \mathbb{R}$. By an application of the Symmetry Principle we can extend $U$ to a harmonic function in $\mathbb{C}$ (which we also denote by $U$ ).

Repeating steps $1-3$ of the proof of Theorem 1' we obtain

$$
\left|U\left(r e^{i \theta}\right)\right| \leq \exp (o(r)) \quad \text { for } r=\frac{r_{k}}{2} \rightarrow \infty .
$$

Suppose that we have shown

$$
|U(z)|=O\left(|z|^{q+3}\right) \quad \text { as } z \rightarrow \infty, \quad|\operatorname{Im} z|<\frac{H}{2} .
$$

Then using the methods of steps 5 and 6 in the proof of Theorem 1' we get $U(z)=\operatorname{Im} P(z)$, where $P$ is a real polynomial of degree at most $q+4$. Noting that the convergence of the first integral in (7) implies

$$
\int_{-\infty}^{\infty} \frac{|U(x+i H)|}{1+|x|^{q+1}} d x<\infty
$$

we obtain that the degree of $P$ is not greater than $q$ and hence the assertion of Theorem 2 holds. Now, it only remains to establish (41).

Set

(43) $v(z)=U(z)-\int_{-\infty}^{\infty} P_{q}(x+i(H-y), t) U(t+i H) d t, \quad z=x+i y, y<H$.

The integral on the right-hand side converges by (42). Therefore, $v$ is harmonic in $\{z: \operatorname{Im} z<H\}$ and continuous in $\{z: \operatorname{Im} z \leq H\}$, if we define $v(x+i H):=0$ for $x \in \mathbb{R}$. Hence $v$ can be extended to a harmonic function in $\mathbb{C}$ (which we also denote by $v$ ).

Now, we show that there exists a sequence $\left\{R_{k}\right\}, R_{k} \rightarrow \infty$, such that

$$
\left|v\left(r e^{i \theta}\right)\right| \leq \exp (o(r)) \quad \text { for } r=R_{k} \rightarrow \infty .
$$

Set $\tilde{v}(\zeta):=v(\zeta+i H)$. Then, by (43) with $\rho_{k}:=r_{k} / 2-H$,

$$
\begin{aligned}
\int_{-\pi}^{0}\left|\tilde{v}\left(\rho_{k} e^{i \theta}\right)\right||\sin \theta| d \theta \leq & \int_{-\pi}^{0}\left|U\left(\rho_{k} e^{i \theta}+i H\right)\right||\sin \theta| d \theta \\
& +\int_{-\pi}^{0}\left(\int_{-\infty}^{\infty}\left|P_{q}\left(\rho_{k} e^{-i \theta}, t\right)\right||U(t+i H)| d t\right)|\sin \theta| d \theta \\
=: & I_{1}+I_{2} .
\end{aligned}
$$

From (40) we obtain $I_{1} \leq \exp \left(o\left(\rho_{k}\right)\right)$ as $\rho_{k} \rightarrow \infty$. Using Fubini's Theorem and the estimate (12) we get $I_{2} \leq \exp \left(o\left(\rho_{k}\right)\right)$ as $\rho_{k} \rightarrow \infty$ by an argument similiar to that at the end of step 1 in the proof of Theorem 1'. By symmetry,

$$
\int_{0}^{\pi}\left|\tilde{v}\left(\rho_{k} e^{i \theta}\right)\right| \sin \theta d \theta \leq \exp \left(o\left(\rho_{k}\right)\right) \quad \text { as } \rho_{k} \rightarrow \infty
$$


In the same fashion as in step 3 of the proof of Theorem 1' we obtain from (45) that

$$
|\tilde{v}(z)| \leq \exp (o(|z|)) \quad \text { for }|z|=\frac{\rho_{k}}{2} \rightarrow \infty .
$$

Since $|v|$ is a subharmonic function in $\mathbb{C},(44)$ holds for $R_{k}=\rho_{k} / 2-H$.

Now, we show that

$$
|v(x)| \leq O\left(|x|^{q+2}\right) \quad \text { for } x \rightarrow \infty, x \in \mathbb{R} .
$$

Since $U(x)=0$ for $x \in \mathbb{R}$, it follows from (43) and estimate (12) that for $|x|$ sufficiently large

$$
\begin{aligned}
|v(x)| & \leq \int_{-\infty}^{\infty}\left|P_{q}(x+i H, t)\right||U(t+i H)| d t \\
& \leq D_{q}|x|^{q} \int_{-\infty}^{\infty} \frac{H}{(x-t)^{2}+H^{2}} \frac{1}{(1+|t|)^{q-1}}|U(t+i H)| d t \\
& \leq D_{q, H}|x|^{q}\left(\int_{|t|<1}|U(t+i H)| d t+\int_{|t| \geq 1} \frac{|U(t+i H)|}{1+|t|^{q+1}} \frac{2 t^{2}}{(x-t)^{2}+H^{2}} d t\right) \\
& =O\left(|x|^{q}\right)+D_{q, H}|x|^{q} \max _{|t| \geq 1} \frac{2 t^{2}}{(x-t)^{2}+H^{2}} \int_{|t| \geq 1} \frac{|U(t+i H)|}{1+|t|^{q+1}} d t \\
& =O\left(|x|^{q+2}\right), \quad \text { as }|x| \rightarrow \infty .
\end{aligned}
$$

Therefore, (46) is true.

Now, let $V$ be an entire function such that $\operatorname{Re} V(z)=v(z)$. Define

$$
F(z):= \begin{cases}e^{V(z)-A z^{q+2}} & \text { if } q \text { is even, } \\ e^{V(z)-A z^{q+3}} & \text { if } q \text { is odd }\end{cases}
$$

where $A>0$ is a constant. It is evident from (46) that $F$ is bounded on the boundary of the strip $S:=\{z \in \mathbb{C}: 0<\operatorname{Im} z<H\}$ if $A$ is large enough.

From (44) it follows that

$$
|F(z)| \leq \exp (\exp (o(|z|))), \quad \text { for }|z|=R_{k} \rightarrow \infty, z \in S .
$$

An application of the Phragmén-Lindelöf Principle for a strip (see, e.g. [9, p. 40]) shows that $F$ is bounded on $S$. This implies

$$
v(z)=\log |F(z)|+O\left(|z|^{q+3}\right) \leq O\left(|z|^{q+3}\right), \quad \text { as } z \rightarrow \infty, z \in S .
$$

Similarly, by replacing $V$ with $-V$ in (47), we get

$$
-v(z) \leq O\left(|z|^{q+3}\right), \quad \text { as } z \rightarrow \infty, z \in S .
$$

From (48) and (49) we conclude

$$
|v(z)|=O\left(|z|^{q+3}\right), \quad \text { as } z \rightarrow \infty, z \in S .
$$


As in the proof of (46) we show

$$
\begin{array}{r}
\left|\int_{-\infty}^{\infty} P_{q}(x+i(H-y), t) U(t+i H) d t\right|=O\left(|z|^{q+2}\right), \\
\text { as } z \rightarrow \infty, 0<\operatorname{Im} z<\frac{H}{2} .
\end{array}
$$

Then (41) follows from (43), (50) and (51).

\section{Proof of Theorem 1}

We need the following well-known result for the representation of a function defined in a strip by a Poisson integral.

Lemma 4. Let $v$ be a function harmonic in a strip $S_{h}:=\{z \in \mathbb{C}: 0<\operatorname{Im} z<h\}$ and continuous in its closure $\bar{S}_{h}$.

If there exist two sequences $x_{j}^{+} \rightarrow+\infty$ and $x_{j}^{-} \rightarrow-\infty$ such that

$$
\int_{0}^{h}|v(x+i y)| \sin \frac{\pi y}{h} d y=o\left(e^{\pi|x| / h}\right), \quad \text { as } x=x_{j}^{ \pm}, j \rightarrow \infty
$$

and if

$$
\int_{-\infty}^{\infty}(|v(x)|+|v(x+i h)|) e^{-\pi|x| / h} d x<\infty
$$

then $v$ admits the representation

$$
\begin{array}{r}
v(z)=\frac{\sin \frac{\pi y}{h}}{2 h} \int_{-\infty}^{\infty} \frac{v(t) d t}{\cosh \frac{\pi(x-t)}{h}-\cos \frac{\pi y}{h}} \\
+\frac{\sin \frac{\pi y}{h}}{2 h} \int_{-\infty}^{\infty} \frac{v(t+i h) d t}{\cosh \frac{\pi(x-t)}{h}+\cos \frac{\pi y}{h}}, \\
z=x+i y \in S_{h} .
\end{array}
$$

Since we could not find a convenient reference we shall give a proof.

Proof. Denote the expression on the right-hand side of (54) by $T(z)$ and let

$$
V(z):=v(z)-T(z) .
$$

It is easy to see that $T(z)$ is harmonic in $S_{h}$, continuous in $\bar{S}_{h}$ and takes the same values as $v(z)$ on $\partial S_{h}$. Indeed, the change of variables $\zeta=e^{\pi z / h}, v(t)=v_{1}\left(e^{\pi t / h}\right)$, $v(t+i h)=v_{1}\left(-e^{\pi t / h}\right)$, reduces $T(z)$ to the Poisson integral of $v_{1}$ for $\mathbb{C}_{+}$.

By the Symmetry Principle the function $V(z)$ can be extended to the whole complex plane $\mathbb{C}$ as a harmonic function (which we also denote it by $V(z)$ ) 
which is odd and $2 h$-periodic with respect to $y=\operatorname{Im} z$. This function can be expanded into the absolutely convergent Fourier series

$$
V(x+i y)=\sum_{k=1}^{\infty} c_{k}(x) \sin \frac{k \pi y}{h},
$$

where

$$
c_{k}(x)=\frac{1}{h} \int_{0}^{h} V(x+i y) \sin \frac{k \pi y}{h} d y, \quad k=1,2, \ldots
$$

Since $V$ satisfies the Laplace equation we get

$$
c_{k}^{\prime \prime}(x)-\left(\frac{k \pi}{h}\right)^{2} c_{k}(x)=0, \quad k=1,2, \ldots
$$

Therefore

$$
c_{k}(x)=c_{1 k} e^{k \pi x / h}+c_{2 k} e^{-k \pi x / h}, \quad k=1,2, \ldots
$$

where $c_{1 k}$ and $c_{2 k}$ are constants not depending on $x$.

On the other hand,

$$
\begin{aligned}
c_{k}(x) & =\frac{1}{h} \int_{0}^{h} v(x+i y) \sin \frac{k \pi y}{h} d y-\frac{1}{h} \int_{0}^{h} T(x+i y) \sin \frac{k \pi y}{h} d y \\
& =: c_{k}^{(1)}(x)-c_{k}^{(2)}(x) .
\end{aligned}
$$

The elementary inequality $|\sin k \tau| \leq k \sin \tau, 0 \leq \tau \leq \pi$, implies

$$
\begin{aligned}
\left|c_{k}^{(1)}(x)\right| & \leq \frac{k}{h} \int_{0}^{h}|v(x+i y)| \sin \frac{\pi y}{h} d y, \\
\left|c_{k}^{(2)}(x)\right| & \leq \frac{k}{h} \int_{0}^{h}|T(x+i y)| \sin \frac{\pi y}{h} d y .
\end{aligned}
$$

Evidently, condition (52) implies

$$
c_{k}^{(1)}(x)=o\left(e^{\pi|x| / h}\right), \quad \text { as } x=x_{j}^{ \pm}, j \rightarrow \infty .
$$

Let us show that

$$
c_{k}^{(2)}(x)=o\left(e^{\pi|x| / h}\right) \quad \text { as }|x| \rightarrow \infty .
$$

Substituting the expression for $T(x+i y)$ and using Fubini's Theorem we obtain

$$
\begin{aligned}
\left|c_{k}^{(2)}(x)\right| \leq & \frac{k}{2 h^{2}} \int_{-\infty}^{\infty}|v(t)|\left(\int_{0}^{h} \frac{\sin ^{2} \frac{\pi y}{h}}{\cosh \frac{\pi(x-t)}{h}-\cos \frac{\pi y}{h}} d y\right) d t \\
& +\frac{k}{2 h^{2}} \int_{-\infty}^{\infty}|v(t+i h)|\left(\int_{0}^{h} \frac{\sin ^{2} \frac{\pi y}{h}}{\cosh \frac{\pi(x-t)}{h}+\cos \frac{\pi y}{h}} d y\right) d t
\end{aligned}
$$


A standard calculation shows

$$
\int_{0}^{h} \frac{\sin ^{2} \frac{\pi y}{h}}{\cosh \frac{\pi(x-t)}{h} \pm \cos \frac{\pi y}{h}} d y=h e^{-\pi|x-t| / h} .
$$

Therefore,

$$
\begin{aligned}
\left|c_{k}^{(2)}(x)\right| & \leq \frac{k}{2 h} \int_{-\infty}^{\infty}(|v(t)|+|v(t+i h)|) e^{-\pi|x-t| / h} d t \\
& =\frac{k}{2 h} e^{\pi|x| / h} \int_{-\infty}^{\infty}(|v(t)|+|v(t+i h)|) e^{-\pi|t| / h} e^{(\pi / h)(|t|-|x|-|x-t|)} d t .
\end{aligned}
$$

Since $\exp ((\pi / h)(|t|-|x|-|x-t|))$ is bounded by 1 and tends to 0 as $|x| \rightarrow \infty$, we obtain (57) from condition (53) with help of the Lebesgue Dominated Convergence Theorem.

Equations (56), (57) and (55) show $c_{k}(x)=0, k=1,2, \ldots$ Hence, $V(z)=0$ and $v(z)=T(z)$.

To prove Theorem 1 it suffices to show that its conditions imply condition (13). Then the assertion of Theorem 1 will follow from Theorem 1'.

By condition (5) of Theorem 1, there exists a constant $C>0$ and a decreasing sequence $\left\{s_{l}\right\}_{l=1}^{\infty} \subset(0,1], \lim _{l \rightarrow \infty} s_{l}=0$, such that

$$
\int_{-\infty}^{\infty} \frac{\left|u\left(x+i s_{l}\right)\right|}{1+|x|^{\alpha}} d x \leq C, \quad l=1,2, \ldots
$$

Clearly, it suffices to show that

$$
\sup _{s_{l}<y<s_{1}} \int_{-\infty}^{\infty} \frac{|u(x+i y)|}{1+|x|^{\alpha}} \leq D, \quad l=1,2, \ldots,
$$

where $D$ does not depend on $l$.

We want to apply Lemma 4 to $v_{l}(z)=u\left(z+i s_{l}\right)$ for $h=h_{l}:=s_{1}-s_{l}, l>1$. It is clear from (58) that $v_{l}$ satisfies (53) of Lemma 4 . To show that (52) of Lemma 4 is also satisfied, we need to apply Corollary 1 to the function $v_{l+1}(z)=u\left(z+i s_{l+1}\right)$. It is easy to see that condition (8) is satisfied with $H=h_{l+1}$. Now we have to show that there exists a sequence $\left\{R_{k}\right\}, R_{k} \rightarrow \infty$, such that

$$
\int_{0}^{\pi} v_{l+1}^{+}\left(r e^{i \theta}\right) \sin \theta d \theta \leq \exp (o(r)) \quad \text { as } r=R_{k} \rightarrow \infty .
$$

Let

$$
Q_{R, s_{l+1}}=\left\{z \in \mathbb{C}:\left|z+i s_{l+1}\right|<R\right\} \cap \mathbb{C}_{+} \quad \text { for } R>s_{l+1} .
$$

Since the function $v_{l+1}^{+}(z)$ is subharmonic in the closure of $Q_{R, s_{l+1}}$ we have

$$
v_{l+1}^{+}(z) \leq \frac{1}{2 \pi} \int_{\partial Q_{R, s_{l+1}}} v_{l+1}^{+}(\zeta) \frac{\partial}{\partial n} G_{Q_{R, s_{l+1}}}(\zeta, z)|d \zeta| \quad \text { for } z \in Q_{R, s_{l+1}},
$$


where $G_{Q_{R, s_{l+1}}}(\zeta, z)$ is the Green function of $Q_{R, s_{l+1}}$ and $\partial / \partial n$ is the derivative in the direction of the inner normal.

Let

$$
K_{R, s_{l+1}}=\left\{z \in \mathbb{C}:\left|z+i s_{l+1}\right|<R, \operatorname{Im} z>-s_{l+1}\right\} .
$$

According to the Principle of Extension of Domains (see [2, p. 3], or in an equivalent form [12, Ch. IV $\S 2]$ ), we have

$$
\frac{\partial}{\partial n} G_{Q_{R, s_{l+1}}}(\zeta, z) \leq \frac{\partial}{\partial n} G_{K_{R, s_{l+1}}}(\zeta, z) \quad \text { for } \zeta \in \partial Q_{R, s_{l+1}} \cap \partial K_{R, s_{l+1}}, z \in Q_{R, s_{l+1}}
$$

Using the well-known explicit expression for the Green function of a half-disc, we get (with the choice $z_{s_{l+1}}=z+i s_{l+1}, \varphi_{s_{l+1}}=\arg z_{s_{l+1}}$ )

$$
\begin{aligned}
\int_{\partial Q_{R, s_{l+1}} \cap \partial K_{R, s_{l+1}}} v_{l+1}^{+}(\zeta) \frac{\partial}{\partial n} G_{Q_{R, s_{l+1}}}(\zeta, z)|d \zeta| \\
\quad \leq \int_{\partial Q_{R, s_{l+1}} \cap \partial K_{R, s_{l+1}}} v_{l+1}^{+}(\zeta) \frac{\partial}{\partial n} G_{K_{R, s_{l+1}}}(\zeta, z)|d \zeta| \\
\quad=\int_{\arcsin \left(s_{l+1} / R\right)}^{\pi-\arcsin \left(s_{l+1} / R\right)} u^{+}\left(R e^{i \theta}\right) \frac{4 R\left|z_{s_{l+1}}\right|\left(R^{2}-\left|z_{s_{l+1}}\right|^{2}\right) \sin \theta \sin \varphi_{s_{l+1}}}{\left|R e^{i \theta}-z_{s_{l+1}}\right|^{2}\left|R e^{-i \theta}-z_{s_{l+1}}\right|^{2}} d \theta \\
\leq \frac{4\left(R+|z|+s_{l+1}\right)^{3}}{\left(R-|z|-s_{l+1}\right)^{3}} \int_{0}^{\pi} u^{+}\left(R e^{i \theta}\right) \sin \theta d \theta
\end{aligned}
$$

Furthermore, since $Q_{R, s_{l+1}} \subset \mathbb{C}_{+}$, we obtain

$$
\frac{\partial}{\partial n} G_{Q_{R, s_{l+1}}}(\zeta, z) \leq \frac{\partial}{\partial n} G_{\mathbb{C}_{+}}(\zeta, z) \quad \text { for } \zeta \in \partial Q_{R, s_{l+1}} \backslash \mathbb{C}_{+}, z \in Q_{R, s_{l+1}}
$$

Hence,

$$
\begin{aligned}
\int_{\partial Q_{R, s_{l+1}} \backslash \mathbb{C}_{+}} v_{l+1}^{+}(\zeta) \frac{\partial}{\partial n} G_{Q_{R, s_{l+1}}}(\zeta, z)|d \zeta| \\
\quad \leq \int_{\partial Q_{R, s_{l+1}} \backslash \mathbb{C}_{+}} v_{l+1}^{+}(\zeta) \frac{\partial}{\partial n} G_{\mathbb{C}_{+}}(\zeta, z)|d \zeta| \\
\quad=\int_{-\sqrt{R^{2}-s_{l+1}^{2}}}^{\sqrt{R^{2}-s_{l+1}}} u^{+}\left(t+i s_{l+1}\right) \frac{2 y}{(x-t)^{2}+y^{2}} d t \\
\leq \int_{-R}^{R} u^{+}\left(t+i s_{l+1}\right) \frac{2 y}{(x-t)^{2}+y^{2}} d t, \quad z=x+i y .
\end{aligned}
$$


Joining (60) and (61) we obtain

$$
\begin{aligned}
& v_{l+1}^{+}\left(r e^{i \varphi}\right) \leq \frac{2\left(R+r+s_{l+1}\right)^{3}}{\pi\left(R-r-s_{l+1}\right)^{3}} \int_{0}^{\pi} u^{+}\left(R e^{i \theta}\right) \sin \theta d \theta \\
&+\frac{1}{\pi} \int_{-R}^{R} u^{+}\left(t+i s_{l+1}\right) \frac{r \sin \varphi}{r^{2}+t^{2}-2 r t \cos \varphi} d t \\
& r e^{i \varphi} \in Q_{R, s_{l+1}} .
\end{aligned}
$$

For $0<r<R-s_{l+1}$ and $0<\varphi<\pi$ we have $r e^{i \varphi} \in Q_{R, s_{l+1}}$. We multiply both sides of $(62)$ by $\sin \varphi$ and integrate with respect to $\varphi$ from 0 to $\pi$. Using the relation $(27)$ we now obtain

$$
\begin{aligned}
\int_{0}^{\pi} v_{l+1}^{+}\left(r e^{i \varphi}\right) \sin \varphi d \varphi \leq & \frac{4\left(R+r+s_{l+1}\right)^{3}}{\pi\left(R-r-s_{l+1}\right)^{3}} \int_{0}^{\pi} u^{+}\left(R e^{i \theta}\right) \sin \theta d \theta \\
& +C_{q}(1+R)^{\alpha} r \int_{-\infty}^{\infty} \frac{u^{+}\left(t+i s_{l+1}\right)}{1+|t|^{\alpha}} d t .
\end{aligned}
$$

Remember that the function $u$ satisfies (B1). Choose $R=r_{k}, r=r_{k} / 2-s_{l+1}$ in (63) where $\left\{r_{k}\right\}$ is the sequence from condition (B1). Then condition (B1) is satisfied for $v_{l+1}^{+}(z)$ with the sequence $\left\{R_{k}\right\}=\left\{r_{k} / 2-s_{l+1}\right\}$ instead of $\left\{r_{k}\right\}$.

Now, by Corollary 1 , the functions $v_{l+1}$ has the representation

$$
\begin{array}{r}
v_{l+1}(z)=\int_{-\infty}^{\infty} P_{q}(z, t) u\left(t+i s_{l+1}\right) d t+\operatorname{Im} P_{l+1}(z), \\
q=\max \{n \in \mathbb{N} \cup\{0\}, n<\alpha\},
\end{array}
$$

where $P_{l+1}$ is a polynomial of degree at most $q$. Using the representation (64) and the estimate (12) we get

$$
\begin{aligned}
\left|v_{l}(x+i y)\right|= & \left|v_{l+1}\left(x+i y+i\left(s_{l}-s_{l+1}\right)\right)\right| \\
\leq & C_{q}\left(1+|x|+h_{l+1}\right)^{q} \int_{-\infty}^{\infty} \frac{\left|u\left(t+i s_{l+1}\right)\right|}{(1+|t|)^{q-1}} \frac{\left(y+\left(s_{l}-s_{l+1}\right)\right) d t}{(x-t)^{2}+\left(y+\left(s_{l}-s_{l+1}\right)\right)^{2}} \\
& +O\left(|x|^{q}\right) \quad \text { as }|x| \rightarrow \infty, 0<y<h_{l}
\end{aligned}
$$

As in the proof of (46) this shows that $\left|v_{l}(x+i y)\right|=O\left(|x|^{q+2}\right)$ as $|x| \rightarrow \infty$, $0<y<h_{l}$. Hence, $v_{l}$ satisfies condition (52) of Lemma 4. An application of Lemma 4 gives formula (54) for the function $v_{l}$. Dividing both sides by $1+|x|^{\alpha}$, integrating with respect to $x$, and changing the order of integration yields

$$
\begin{aligned}
& \int_{-\infty}^{\infty} \frac{\left|u\left(x+i\left(s_{l}+y\right)\right)\right|}{1+|x|^{\alpha}} d x \leq \int_{-\infty}^{\infty}\left|u\left(t+i s_{l}\right)\right| I_{l}^{+}(t) d t \\
&+\int_{-\infty}^{\infty}\left|u\left(t+i s_{1}\right)\right| I_{l}^{-}(t) d t \\
& 0<y<h_{l} .
\end{aligned}
$$


where

$$
I_{l}^{ \pm}(t)=\frac{\sin \frac{\pi y}{h_{l}}}{2 h_{l}} \int_{-\infty}^{\infty} \frac{d x}{\left(\cosh \frac{\pi(x-t)}{h_{l}} \pm \cos \frac{\pi y}{h_{l}}\right)\left(1+|x|^{\alpha}\right)} .
$$

We now estimate $I_{l}^{ \pm}(t)$. To do so we write

$$
I_{l}^{ \pm}(t)=I_{1, l}^{ \pm}(t)+I_{2, l}^{ \pm}(t)
$$

where

$$
\begin{aligned}
I_{1, l}^{ \pm}(t) & :=\frac{\sin \frac{\pi y}{h_{l}}}{2 h_{l}} \int_{|x|>|t| / 2} \frac{d x}{\left(\cosh \frac{\pi(x-t)}{h_{l}} \pm \cos \frac{\pi y}{h_{l}}\right)\left(1+|x|^{\alpha}\right)}, \\
I_{2, l}^{ \pm}(t) & :=\frac{\sin \frac{\pi y}{h_{l}}}{2 h_{l}} \int_{|x|<|t| / 2} \frac{d x}{\left(\cosh \frac{\pi(x-t)}{h_{l}} \pm \cos \frac{\pi y}{h_{l}}\right)\left(1+|x|^{\alpha}\right)} .
\end{aligned}
$$

Evidently,

$$
\begin{aligned}
I_{1, l}^{ \pm}(t) & \leq \frac{1}{1+\left|\frac{t}{2}\right|^{\alpha}} \frac{\sin \frac{\pi y}{h_{l}}}{2 h_{l}} \int_{-\infty}^{\infty} \frac{d x}{\cosh \frac{\pi(x-t)}{h_{l}} \pm \cos \frac{\pi y}{h_{l}}} \\
I_{2, l}^{ \pm}(t) & \leq \frac{1}{2 h_{l}} \int_{|x|<|t| / 2} \frac{d x}{\cosh \frac{\pi(x-t)}{h_{l}}-1} \\
& \leq \frac{1}{2 h_{l}} \frac{|t|}{\cosh \frac{\pi t}{2 h_{l}}-1} .
\end{aligned}
$$

Choosing $n \in \mathbb{N}$ in such a way that $2 n>\alpha+1$ and using the well-known inequality $\cosh \tau-1 \geq \tau^{2 n} /(2 n)$ ! we obtain

$$
I_{2, l}^{ \pm}(t) \leq \frac{|t|}{4 h_{l}} \frac{(2 n) !\left(2 h_{l}\right)^{2 n}}{(\pi|t|)^{2 n}} \leq \frac{D_{1}}{1+|t|^{\alpha}} \quad \text { for }|t| \geq 1
$$

where $D_{1}$ does not depend on $l$ (remember that $h_{l}<1$ ).

An application of Lemma 4 with $v(z) \equiv 1$ yields the identity

$$
1=\frac{\sin \frac{\pi y}{h_{l}}}{2 h_{l}}\left(\int_{-\infty}^{\infty} \frac{d x}{\cosh \frac{\pi(x-t)}{h_{l}}-\cos \frac{\pi y}{h_{l}}}+\int_{-\infty}^{\infty} \frac{d x}{\cosh \frac{\pi(x-t)}{h_{l}}+\cos \frac{\pi y}{h_{l}}}\right) .
$$

Both integrals in the right hand side are positive. Hence we conclude that

$$
I_{1, l}^{ \pm}(t) \leq \frac{D_{2}}{1+|t|^{\alpha}}, \quad \text { for } t \in \mathbb{R}
$$

and, moreover,

$$
I_{l}^{ \pm}(t)=I_{1, l}^{ \pm}(t)+I_{2, l}^{ \pm}(t) \leq 1 \quad \text { for } t \in \mathbb{R} .
$$

Using this and (66) we conclude that

$$
I_{l}^{ \pm}(t) \leq \frac{D_{3}}{1+|t|^{\alpha}} \quad \text { for } t \in \mathbb{R}
$$


where $D_{3}$ does not depend on $l$ and $t$.

Substituting this estimate into (65) we obtain

$$
\begin{aligned}
\int_{-\infty}^{\infty} \frac{\left|u\left(x+i s_{l}+i y\right)\right|}{1+|x|^{\alpha}} d x & \leq D_{3} \int_{-\infty}^{\infty} \frac{\left|u\left(t+i s_{l}\right)\right|+\left|u\left(t+i s_{1}\right)\right|}{1+|t|^{\alpha}} d t \\
& \leq 2 D_{3} C, \quad 0<y<s_{1}-s_{l},
\end{aligned}
$$

where $C$ is the constant from (58). Hence, (59) follows.

Acknowledgement. The authors express their deep gratitude to Alexander Iljinskii, Alexander Ulanovskii and Natalya Zheltukhina for careful reading of the manuscript and for valuable remarks and to the referee and copy editor for numerous suggestions substantially improving the presentation of the paper.

\section{References}

1. N. I. Akhiezer, Lectures on Integral Transforms, AMS, Providence, R. I., 1988.

2. T. Carleman, Sur les fonctions inverses des fonctions entiéres d'ordre fini, Ark. Mat. 15 (1921) no. 10, 1-7.

3. S. Gergün, I. V. Ostrovskii and A. Ulanovskii, On the Titchmarsh convolution theorem, C. R. Acad. Sci. Paris, Sér. I Math. 331 (2000), 41-46.

4. $\_$On the Titchmarsh convolution theorem, Ark. Mat. 40 (2002) no. 1, 55-71.

5. A. Goldberg and I. V. Ostrovskii, The Value Distribution of Meromorphic Functions, Nauka, Moscow, 1970 (in Russian).

6. N. V. Govorov, Riemann's Boundary Problem with Infinite Index, Birkhäuser Verlag, Basel-Boston, 1994.

7. P. Koosis, Introduction to $H_{p}$ Spaces, Cambridge University Press, 1998.

8. _ The Logarithmic Integral, I, Cambridge University Press, 1998.

9. B. Ya. Levin, Lectures on Entire Functions, AMS, Providence, R.I., 1996.

10. _ Distribution of Zeros of Entire Functions, AMS, Providence, R.I., 1980.

11. R. Nevanlinna, Über die Eigenschaften meromorpher Funktionen in einem Winkelraum, Acta Soc. Sci. Fenn. 50 (1925) no. 12, 1-45.

12. __ Analytic Functions, Springer, Berlin-Heidelberg-New York, 1970.

Seçil Gergün $\quad$ E-MAIL: gergun@fen.bilkent.edu.tr AdDress: Department of Mathematics, Bilkent University, 06800 Bilkent, Ankara, Turkey

Iossif V. Ostrovskii E-MAIL: iossif@fen.bilkent.edu.tr AdDress: Department of Mathematics, Bilkent University, 06800 Bilkent, Ankara, Turkey; Institute for Low Temperature Physics and Engineering, ${ }^{7} 7$ Lenin ave, 61103 Kharkov, Ukraine 